\title{
Комплексная технология о фрактальности среды под наземной лавой
}

\author{
Смагличенко Т.А. ${ }^{1}$, Смагличенко А.В. ${ }^{2,3}$, Саянкина М.К. ${ }^{1}$ \\ ${ }^{1}$ Институт проблем нефти и газа ИПНГ РАН, Москва, tasmaglichr@gmail.com; \\ msayankina@gmail.com \\ ${ }^{2}$ Институт проблем управления им. В.А. Трапезникова ИПУ РАН, Москва, losaeylin@gmail.com \\ ${ }^{3}$ Институт сейсмологии и геодинамики КФУ им В.И. Вернадского, Симферополь, losaеylin@gтаil.com
}

Аннотация. В статье анализируются фрактальные свойства геологической среды на юге Исландии, по результатам практического применения междисциплинарной, так называемой «рамочной» технологии, поиска конфигураций устойчивого линейного роста скорости сейсмической волны с глубиной. В основу технологии положен вычислительный метод нахождения градиентной 1D минимальной модели, также разработанный авторами. Отличием метода от существующих подходов 1D моделирования - в использовании известного разложения Тейлора относительно только двух параметров линейного градиента. Исследована среда, включающая видимую Тьоурсау лаву, извергавшуюся в голоцене. Отметим, лавовые потоки расположены в непосредственной близости от гидротермальных районов производства энергии. Применение технологии выявило градиентную фрактальность, а именно сейсмические свойства, оцененные для структуры к западу от вулкана Хекла в диапазоне глубин 1-4 км, оказались идентичны свойствам структур, расположенных на 2 км глубже в низменности между реками Олфуса и Тьоурсау.

Ключевые слова: 1D минимальная модель, метод наименьших квадратов, Тьоурсау лава, южная сейсмическая исландская зона, комплексный подход, фрактальность среды, гидротермальные поля.

\section{Complex technology about fractality of the medium under the ground lava}

\author{
Smaglichenko T.A. ${ }^{1}$, Smaglichenko A.V. ${ }^{2,3}$, Sayankina M.K. ${ }^{1}$ \\ ${ }^{1}$ Research Oil and Gas Institute IPNG RAS, Moscow,tasmaglichr@gmail.com; msayankina@gmail.com \\ ${ }^{2}$ V.A. Trapeznikov Institute of Control Sciences IPU RAS, Moscow,losaeylin@gmail.com \\ ${ }^{3}$ Institute of Seismology and Geodynamics V.I. VernadskyCFU, Simferopol, losaeylin@gmail.com
}

\begin{abstract}
Fractal properties of a geological medium in the south of Iceland have been analyzed in this article. Results have been obtained by practical application of interdisciplinary, so-called «framework» technology performing the search of configurations of stable linear growth of the seismic wave velocity with depth. The technology is based on the computational method for finding the gradient 1D minimum model that is also developed by the authors. The difference of the method from approaches of 1D modeling is in using the well-known Taylor expansion for only two parameters of a linear gradient. The medium that includes the visible Thjorsa lava erupted in the Holocene has been investigated. It should be noted that lava flows are located close to hydrothermal areas of energy production. An application of the technology revealed a gradient fractality, namely, seismic properties, estimated for the structure to the west of the Hekla volcano in the depth range of $1-4 \mathrm{~km}$, are identical to the properties of structures located deeper on $2 \mathrm{~km}$ in the lowland between the Olfus and Thjorsa rivers.
\end{abstract}

Key words: 1D minimum model, least-square method, Thjorsa lava, south Iceland seismic zone, a complex approach, fractality of a medium, hydrothermal fields.

\section{Введение}

1-D минимальные однородно-слоистые модели были изначально предложены для улучшения качества решения инверсионной проблемы, связывающей одновременно два определяемых параметра: скорость сейсмической волны и координаты гипоцентров (Kissling, 1988, Husen et al., 2003). Затем они хорошо зарекомендовали себя для преодоления систематической ошибки в исходных данных вступлений сейсмических волн (Mauer et al., 2010), а также для более точного распределения гипоцентров на локальном и региональном уровнях (Husen et al., 2011; Kianimehr et al., 2018).

Следует отметить, что для некоторых регионов результаты взрывного профилирования (Bjarnason et al., 1993; Miller and Xia, 1996) показывают близость моделей к линейным градиентам. В то же время надо понимать, что это не повсеместное явление. Так, на Фенноскандинавском щите в 
пробуренных на значительную глубину скважинах не установлена прямая связь с градиентным распределением скорости (Шаров, 2017). Однако присутствие отдельных сегментов градиентного поведения при общей тенденции региона к мозаично-блоковой модели с фрактальной структурой возможно (Шаров, 2017).

Продвижением в теории инверсионных методов явилась разработка метода нахождения 1D минимальной градиентной модели (Smaglichenko et al., 2016) и на его основе создание технологии поиска площадей, в рамках конфигураций которых градиентная модель является устойчивой в смысле статистических оценок по отношению к произвольному множеству других 1D моделей (Smaglichenko et al., 2018). В статье мы сопоставляем найденные модели для районов, расположение которых совпало с территорией наземных лавовых потоков на юге Исландии в системе реки Тьоурсау. Согласно (Гептнер, 2014), это подушечные лавы, сформированные в плейстоцене, а также голоценовые лавовые покровы. Наземные лавовые потоки находятся в одной зоне с промышленным объектом по добыче газа-диоксида углерода в окрестности вулкана Гримснес, с геотермальной электростанцией в южной части зоны вулкана Хенгиль (Бьорнссон, 2007). Более того, на лаве построены города Селфосс, Эйрарбакки и Стокксейри.

\section{1. «Рамочная» технология на основе метода почска $1 D$ минимальной градиентной модели}

Метод поиска 1D минимальной градиентной модели базируется на применении разложения Тейлора к функции времени пробега сейсмической волны, распространяющейся от источника к приёмнику. Применение разложения Тейлора в сейсмологии впервые было предложено Л. Гейгером в 1910 г., когда в качестве известных входных параметров использовались координаты и начальное время стартового гипоцентра, а неизвестными были четыре параметра поправок к этим стартовым значениям (Geiger, 1910). В 1976 г. К. Аки по аналогии сформулировал проблему, но неизвестными параметрами были как поправки к гипоцентрам, так и множество 3D вариаций скорости по отношению к стартовой модели (Aki and Lee, 1976). В 1988 г. Э. Кисслинг ввёл концепцию минимальной однородно-слоистой 1D модели, упростив 3D минимизацию путём нахождения поправок для стартовой слоистой модели (Kissling, 1988). В 2016 г. была опубликована теория по определению минимальной градиентной 1D модели (Smaglichenko et al., 2016). Вычислительное преимущество градиентной модели перед слоистым аналогом в том, что значительно уменьшается число определяемых параметров модели. А именно, вместо многочисленных мощностей слоёв и значений скорости в них определяются только два параметра градиента: значение скорости на поверхности и значение наклона линейной функции. Это позволяет сузить класс допустимых решений с целью преодоления проблемы многозначности решений в смысле метода наименьших квадратов.

Метод был протестирован для данных неглубоких (до 8 км глубины) 203 землетрясений, зарегистрированных Метеорологической Службой Исландии с 4.01.2001 по 28.10.2001 в юго-западной вулканической зоне Исландии. В качестве начальных моделей использовались две модели, имеющие различные параметры линейных градиентов. Первая модель соответствовала SIL модели, полученной в результате проведения взрывных работ на сейсмических профилях на юге Исландии в 1990 г. (Bjarnason et al., 1993). Эта модель до сих пор является общепринятой используемой моделью для материковой части Исландии и до глубины 8 км была аппроксимирована моделью, имеющей параметры $\mathrm{V}(\mathrm{z})=\mathrm{a}+\mathrm{b} * \mathrm{z}$, где $\mathrm{a}=3.926, \mathrm{~b}=0.479$. Вторая модель была найдена по данным землетрясений, очаги которых расположены в море на северо-востоке Исландии в разломной зоне Тьёрнес (Smaglichenko et al., 2009), её параметры: $a=5.9895, b=0.0579$. Метод был применён к обеим стартовым моделям, и в результате были построены минимальные модели, которые практически совпали. Таким образом, с какой бы стартовой модели не началось применение метода, в результате получается одна и та же минимальная модель, имеющая параметры $\mathrm{a}=4.147, \mathrm{~b}=0.441$ и статистические оценки mean $=-0.0013 ; \mathrm{std}=0.0371 /$. Тот факт, что параметры исходных стартовых моделей сильно отличались, свидетельствует об устойчивости метода по отношению к большим колебаниям в значениях параметров начальных моделей. 
Разработанная технология для поиска геотермального поля основана на междисциплинарном подходе, который объединяет численные методы, географические и геодинамические данные, а также понятия теории хаоса (Smaglichenko et al., 2018). Множество исходных природных сейсмических источников и станций, принявших сигналы, делится на подмножества, соответствующие географическим компонентам. Различные районы могут включать высокогорья, низменности, области вулканов. Границы районов могут быть выбраны с учетом местоположения реки, линий разломов. Для каждого выбранного района применяется разработанный метод поиска минимальной линейной градиентной модели, оценивающий линейную функцию скорости сейсмической волны $\mathrm{V}(\mathrm{z})$, которая зависит только от глубины z. Модель считается построенной в рамках выбранной конфигурации среды, если удовлетворены разработанные критерии для получения устойчивых оценок.

\section{2. Минимальные градиентные модели для районов в области наземной лавы Tъоурсау}

Обработка данных нескольких тысяч микроземлетрясений, зарегистрированных в южной исландской сейсмической зоне с 2.02.1991 по 31.12.1991, позволила установить, что основная часть событий, имеющая ошибку гипоцентров в пределах допустимой нормы, распределена до 6 км глубины. Было выявлено несколько районов, которые имели устойчивую минимальную градиентную модель. В данной статье мы исследуем три района, на поверхности которых расположена видимая застывшая лава, являющаяся следствием извержения кратеров вулкана Ватнаолдур в голоцене в центральной Исландии (Hjartarson, 1994). Рисунок 1 показывает район I близ города Эйрарбакки; район II, прилегающий к западному побережью реки Тьоурсау; район III к западу от вулкана Хекла.

Вычисленные градиенты линейного увеличения скорости продольной волны с глубиной совпали для районов I и II, параметры градиентов были определены как $\mathrm{a}=4.7, \mathrm{~b}=0.16$. Для района III получены следующие значения: $\mathrm{a}=4.96, \mathrm{~b}=0.18$. По этим данным ближе к поверхности более низкие скорости могут быть приурочены к районам I и II, расположенным в низменности. Они объясняются присутствием рек и близостью к океану. Микросейсмичность здесь на порядки сильнее, чем в районе III, расположенном севернее ближе к горной местности вулкана Хекла (высота 1.491 м) и характеризующемся в целом повышением уровня моря. Детальный анализ конкретных значений, вы-

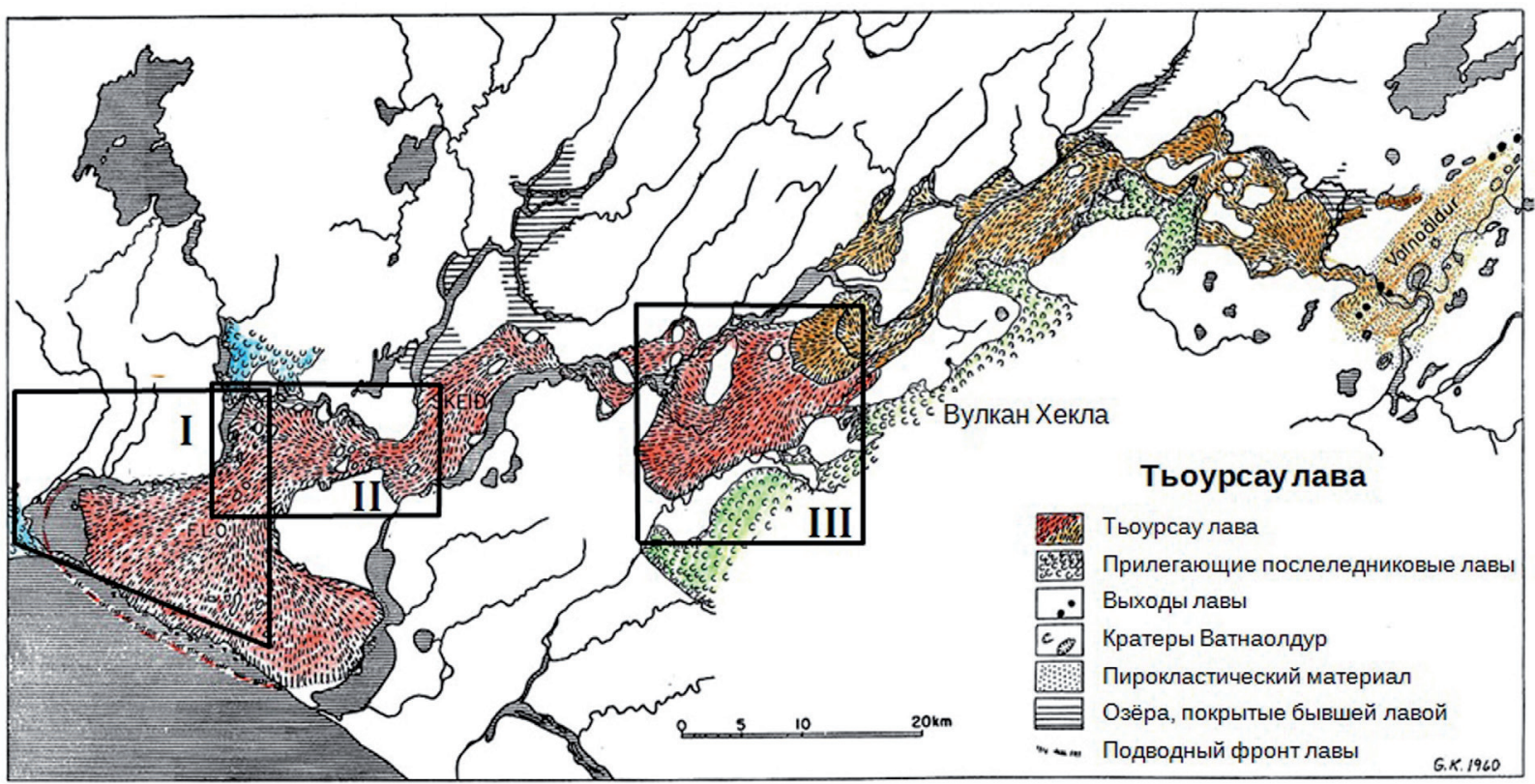

Рис. 1. Наземное распределение Тьоурсау лавы и прилегающие послеледниковые лавовые поля. Данные Гудмундур Кьяртанссон модифицированы Арни Хьяртарсон (Hjartarson, 1988). Выявленные технологией районы I, II, III обозначены сплошной линией.

Fig. 1. Distribution of Thjorsa Lava covering lowlands and adjacent postglacial lava fields. Modified after Guðmundur Kjartansson by Árni Hjartarson (Hjartarson, 1988). Subareas revealed by the technology are noted by a solid line. 


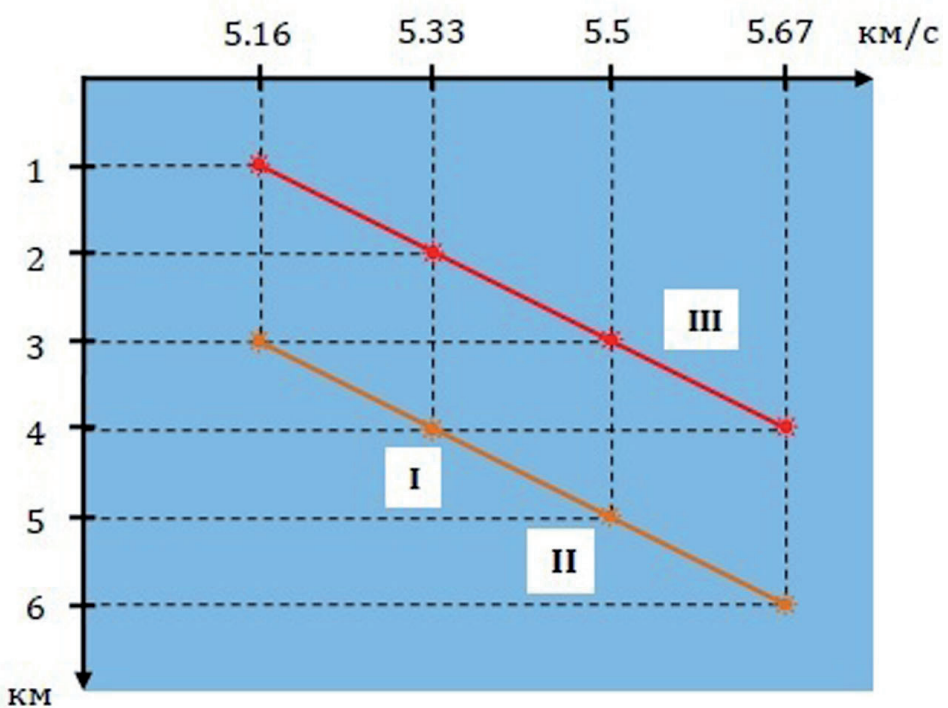

Рис. 2. Оцененные значения скорости продольной волны, характеризующие фрактальные структуры для районов I, II и III.

Fig. 2. Estimated values of P-wave velocity that characterize fractal structures for subareas I, II and III.

численных для разных глубин в соответствии с найденными двумя основными линейными функциями, показал, что строение коры для районов I и II в диапазоне глубин 3-6 км повторяется для района III ближе к поверхности (диапазон глубин 1-4 км). Рисунок 2 иллюстрирует градиентную фрактальность для этих районов.

\section{Заключение}

Предыдущие исследования показали, что применение комплексной междисциплинарной технологии может дать достаточно интересные сведения о подземных структурах и имеет перспективы для поисковых задач (Смагличенко и др., 2017: Smaglichenko et al., 2018). Результаты, продемонстрированные в данной статье, позволили увидеть фрактальность, проявляющуюся в смещении одинаковых структур по глубине на различных участках среды, связанных одним и тем же геологическим процессом. Это расширяет возможности детального изучения площадей с помощью рассматриваемого технологического «инструмента» - 1D минимальной градиентной модели.

Работа выполнена в рамках тем НИР №0139-2019-0009, № 10.331-17, № 5.6370.2017/БЧ

\section{Литература}

1. Бьорнссон С. Развитие геотермальной энергетики и исследования в Исландии: Пер. с исланд. Рейкьявик: Национальный Энергетический Комитет и Министерство промышленности и торговли Исландии. 2007. 39 с.

2. Гептнер А.Р. Вулканогенно-осадочный литогенез в наземной рифтовой зоне Исландии. М.: ГЕОС. 2014. $236 \mathrm{c}$.

3. Смагличенко Т.А., Чигарев Б.Н., Смагличенко А.В. Несмещенная линейная оценка сейсмической скорости при различных температурных режимах геотермальных площадей // Актуальные проблемы нефти и газа. 2017. 2(17). $8 \mathrm{c}$.

4. Шаров Н.В. Литосфера Северной Европы по сейсмическим данным. Петрозаводск: Карельский научный центр РАН. 2017. 173 с.

5. Aki K., Lee W.K.H. Determination of Three-Dimensional Velocity Anomalies under a Seismic Array using First, P-Arrival Times from Local Earthquakes: 1. A Homogeneous Initial Model // Journal of Geophysical Research. 1976. V. 81(23). P. 4381-4399.

6. Bjarnason I.Th., Menke W., Flovenz O.G., Caress D. Tomographic image of the Mid-Atlantic Plate Boundary in Southwestern Iceland // Journal of Geophysical Research. 1993. V. 98(B4). P. 6607-6622.

7. Geiger L. Herdbestimmung bei Erdbeben aus den Ankunftszeiten // Nachrichten von der Koniglichen Gesellschaft der Wissenschaften zu Gottingen. 1910. P. 331-349.

8. Hjartarson A. Bjórsárhraunið mikla - stærsta nútímahraun jarðar // Náttúrufræðingurinn. 1988. V. 58. P. 1-16.

9. Hjartarson A. Environmental changes in Iceland following the great Thjorsa Lava Eruption $780014 \mathrm{C}$ years BP / In: Stцtter J, Wilhelm F, editors. Environmental change in Iceland. Munchen: Geobuch. 1994. P. 147-155. 
10. Husen S. Kissling E. Deichmann N. Wiemer S. Giardini D. Baer M. Probabilistic earthquake location in complex three-dimensional velocity models: application to Switzerland // Journal of Geophysical Research. 2003. V. 108(B2). 26 p.

11. Husen S., Kissling E., Clinton J. Local and Regional Minimum 1D Models for Earthquake Location and Data Quality Assessment in Complex Tectonic Regions: Application to Switzerland // Swiss Journal of Geosciences. 2011. V. 104(3). P. 455-469.

12. Kissling E. Geotomography with local earthquake data // Reviews of Geophysics. 1988. V. 26. P. 659-698.

13. Maurer V., Kissling E., Husen S., Quintero R. Detection of systematic errors in travel-time data using a minimum 1D model: application to Costa Rica seismic tomography // Bulletin of the Seismological Society of America. 2010. V. 100. P. 629-639.

14. .Miller R.D., Xia J. Large near-surface velocity gradients on shallow seismic reflection data // Geophysics. 1998. V. 63(4). P. 1348-1356.

15. Kianimehr H., Kissling E, Yaminifard F., Tatar M. Regional minimum 1-D P-wave velocity model for a new seismicity catalogue with precise and consistent earthquake locations in southern Iran // Journal of Seismology. 2018. V. 22(6) P. 1529-1547.

16. Smaglichenko T., Jacoby W., Fedorova T., Wallner H. Stable Estimate of Velocity Anomalies around Grimsey Lineament (Tjornes Fracture Zone, Iceland) with Differentiated Tomography / In: Arabelos DN, Tscherning CC, editors. EGU General Assembly Conference Abstracts. 2009. V. 11. P. 11-1332.

17. Smaglichenko T.A., Bjarnason I.Th., Smaglichenko A.V., Jacoby W.R. Method to find the minimum 1-D linear gradient model for seismic tomography // Fundamenta Informaticae. 2016. V. 146(2). P. 211-217.

18. Smaglichenko T.A., Smaglichenko A.V., Zelinka I., Chigarev B. Seismic attractor can assist in finding of geothermal area? // International Journal of Parallel, Emergent and Distributed Systems. 2018. V. 33 (5). P. 503-512. 